\title{
3-DIMENSIONAL GEOLOGICAL MAPPING AND MODELING ACTIVITIES AT THE GEOLOGICAL SURVEY OF NORWAY
}

\author{
A.Jarna, A. Bang-Kittilsen, C.Haase, I.H.C. Henderson, F. Høgaas, S.Iversen, A.Seither \\ Norges geologiske undersøkelse (Geological Survey of Norway), Leiv Eirikssons vei 39, 7040, Trondheim, Norway \\ alexandra.jarna@ngu.no
}

KEYWORDS: 3D geology, Geometric modelling, Boreholes, DTM, Geological maps, LiDAR, Geophysics, Urban geology, 3D subsurface modelling

\begin{abstract}
:
Geology and all geological structures are three-dimensional in space. Geology can be easily shown as four-dimensional when time is considered. Therefore GIS, databases, and 3D visualization software are common tools used by geoscientists to view, analyse, create models, interpret and communicate geological data. The NGU (Geological Survey of Norway) is the national institution for the study of bedrock, mineral resources, surficial deposits and groundwater and marine geology. The interest in 3D mapping and modelling has been reflected by the increase of number of groups and researches dealing with 3D in geology within NGU. This paper highlights 3D geological modelling techniques and the usage of these tools in bedrock, geophysics, urban and groundwater studies at NGU, same as visualisation of 3D online. The examples show use of a wide range of data, methods, software and an increased focus on interpretation and communication of geology in 3D. The goal is to gradually expand the geospatial data infrastructure to include 3D data at the same level as $2 \mathrm{D}$.
\end{abstract}

\section{INTRODUCTION}

Showing geology in the full dimensionality started after 1990s closely linked with the progress in development of 3 dimensional (3D) software. Nowadays' software for creating 3D models (GeoVisionary, ER Mapper, 3D Move 3D GeoModeller, Voxel etc.) have enlightened new possibilities in presenting data in Earth Sciences.

The Geological Survey of Norway (NGU), founded in 1858, is the Norwegian government agency responsible for geologic mapping of Norway's land and marine areas, the production and maintenance of national databases and maps and research. In order to take advantage of large datasets and high number of geological observations, GIS techniques are applied to a great extent. The usage of paper and digital maps has become interchangeable.

In Norway, all 2-dimensional (2D) geological data is digital and downloadable for free from the www.ngu.no website. The user group is therefore extremely large and varied, from individual private users to multinational exploration companies. The plan in the future once the $3 \mathrm{D}$ data is created from the existing $2 \mathrm{D}$ data, then this will be available to download from the www.ngu.no website.

2D geological maps remain the most important means of interpretation of geology. 3 dimensional (3D) visualization of data, on the other hand, provides additional information and reduces the limitation of 2 dimensional maps. Geological mapping of the structures was from its very beginning mapped by geologists and creating cross-sections to display third dimension of the landscape.

Advantages of 3D models for better understanding of geology to a large audience are well known (Wijns, Boschetti et al. 2003, Wu, Xu et al. 2005). Interpretation of geological data based on all three dimensions provides better insight on the geological conditions, and more effective communication of complex geological phenomenon and processes. Moreover, 3D modelling contributes to increased interest in geology. 3D inversion applied to geological and geophysical data can provide new attitude in defining the geology.

The methodology of creating 3D model depends on the available data, data size, complexity of the geology, and scale of model. Today's well used methods often imply 3D seismic data, cross-sections or boreholes.

This increase of interest in 3D modelling has been reflected by the increase of number of groups and researches who implement and deal with showing geology in 3D within NGU.

Geology is divided into various fields, each concerns a certain aspect. These aspects include: giving insight into the history of the Earth which is important for better understanding of natural hazards, evaluating water resources, and exploring mineral deposits. Marine geology and mineral resources are examples where also 3D-mapping and presentations have been increasing the last decade. With greater technical possibilities, the focus on offering geology in $3 \mathrm{D}$ to the society increases. Efforts are being made on coordinating 3D activities at NGU to speed up the development of mapping and methods for 3D geology. In this contribution a selection of cases are described and shortly describe some of the main ongoing projects at Geological Survey of Norway, and gives an insight to some of the current activities.

\section{APPLICATIONS}

Some current activities concerning 3D visualization and modelling projects are: 
1- 3D-Norge: this project aims to show the bedrock geology of Norway in $3 \mathrm{D}$.

2- 3D subsurface modelling in urban areas for evaluation of stability and risk analyses, concerning urban development and consequences for groundwater, subsidence and preservation of cultural heritage.

3- Geophysical models describe subsurface structures in terms of geometries and distributions of physical properties (e.g. densities, magnetization, electrical conductivity, seismic velocity, etc.).

4- Representing LiDAR data: the usage of digital elevation models derived from airborne LiDAR and its successful implementation into studying of nowadays young geology.

5- Web solution of presenting 3D data via WebGL API which is a cross-platform.

With the increased activities in 3D mapping, modelling and visualization the need for a standardized workflow and management of models emerges. The geomatics department therefore aims to take effort in involvement to ensure good practice and data management.

Inspired by other geological surveys, like British Geological Survey BGS and Geological Survey of Denmark GEUS , the question of a framework for a geologic 3D multiscale model covering what is mapped in the whole country is continuously discussed. Also, the question of reducing costs by limited the amount of different software and methods is relevant.

The geomatics department has arranged a internal seminar and have started making lists of projects, current activities, software and data on the intranet. A social media site has been started up (Yammer).

\section{MAPPING AND MODELLING AT THE GEOLOGICAL SURWAY}

\subsection{D-Norge: 3D bedrock map of Norway}

One of the main starting points for making a model of the bedrock geology onshore is that the geology is very complex. We therefore need a modelling package that is able to faithfully portray the complex structures in 3 dimensions. To create a fully $3 \mathrm{D}$ bedrock model covering the whole Norway was chosen software 3D MOVE for its user friendliness and ability to model complex structures.

\subsubsection{Surface bedrock mapping}

In the length of this $3 \mathrm{D}$ project it was decided that $1: 250 \mathrm{k}$ national coverage was too detailed to allow a quick production of a nationwide 3D model, therefore the natural scale for the foundation for the 3D model is $1: 2 \mathrm{M}$.

The standard scale for geological maps in Norway is $1: 250 \mathrm{k}$ and more detailed maps constructed at $1: 50 \mathrm{k} .1: 1 \mathrm{~m}$ and $1: 2 \mathrm{~m}$ maps are simplified from the $1: 250 \mathrm{~K}$ compilation. These are $2 \mathrm{D}$ surface maps of the different rock layers constructed from field observations by the geologist. In the present day, the data is collected digitally with android pads in the field using ESRI ArcGIS software. Map production occurs in the office during the winter months using ArcGIS, compiling the newly acquired data and analogue historical data. This is the basis for the 3D input data

\subsubsection{Geological Profiles}

Geological profiles, both $50 \mathrm{~K}$ and $250 \mathrm{~K}$, constructed for the inclusion in the associated map products, have been manually scanned as separate jpeg files. These have been georeferenced in 3D in 3DMOVE.

There is a wide spectrum of quality of the profiles: the $250 \mathrm{~K}$ profiles tend to be of high quality whereas many of the $50 \mathrm{~K}$ profiles are poorly constructed and drawn (Figure 1, Figure 2).

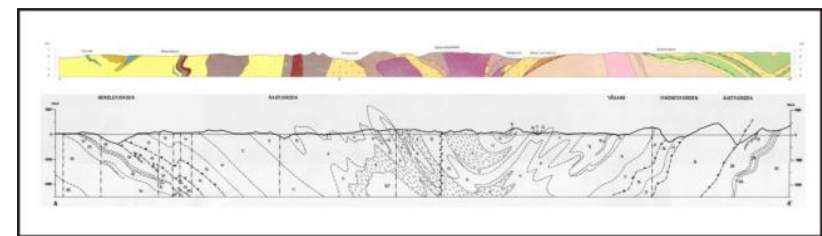

Figure 1. Examples of $250 \mathrm{~K}$ and $50 \mathrm{~K}$ profiles showing the difference in quality.

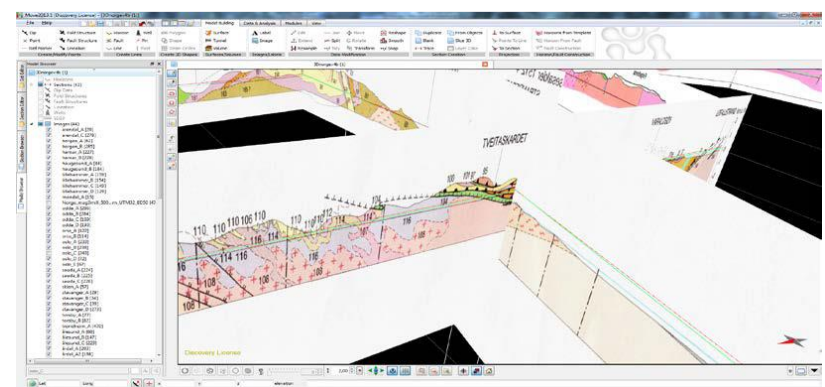

Figure 2: Screen dump from 3DMOVE showing the mismatch between two intersecting $250 \mathrm{~K}$ profiles.

These mismatches (Figure 2) show that the original, analogue profiles, the majority of which were constructed with pencil and paper by the geologist, were wrongly constructed. We need to go back to the original map and make a new profile ! IN reality, the amount of wrongly constructed profiles is low. We have a total of ca. 650 profiles in the national model and, while the work is long from finished, we have perhaps less than $1 \%$ mismatch.

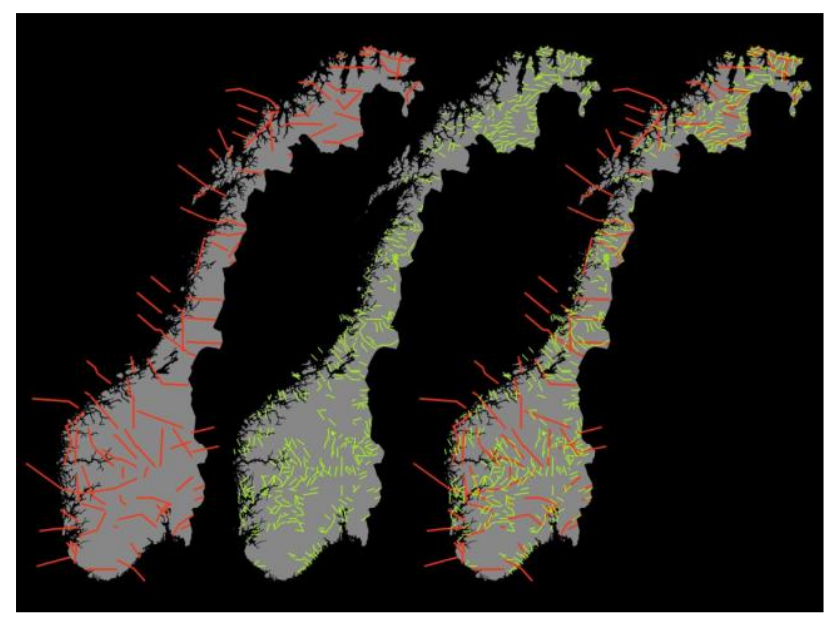

Figure 3: Map of Norway showing the existing 1:250K geological profiles (left in red), the existing 1:50K geological profiles (middle in green) and the combined profiles on the right. 


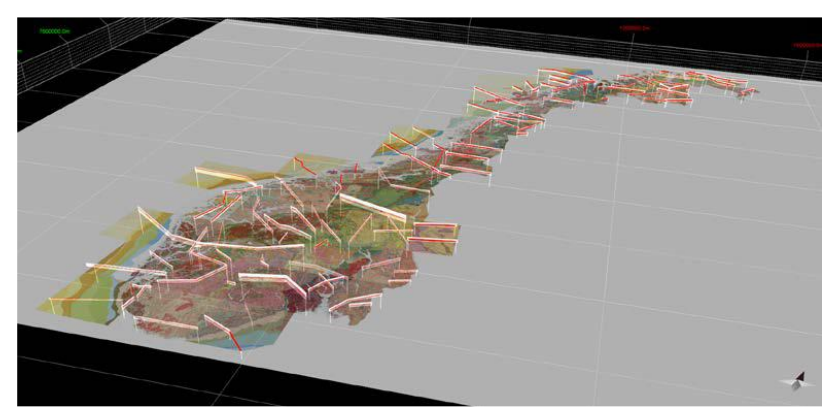

Figure 4: Screen dump from 3DMOVE showing all of the 250K profiles georeferenced in 3D with the bedrock surface geology.

At the moment are data collected and compiled the basis data that are required to begin construction of the $3 \mathrm{D}$ model. This includes, 250k profiles (Figure 4), 50k profiles, 250k surface mapping, the simplified 1:2M tectonostratigraphic. All of the basis data are entered except the $50 \mathrm{~K}$ profiles. this is $20 \%$ finished. Once this is done then the job will start with making 3D shapefiles (line files) and subsequent 3D mesh surfaces.

\subsection{D geophysical modelling}

Geophysical models are basically distributions of physical properties in the subsurface. They aid in the understanding of geological structures, tectonic settings, reservoir identifications and monitoring, archaeological sites, and many more. Usually, the physical properties cannot be measured directly. Instead observables are measured which are related to the properties by physical laws. In order to be used in geophysical modeling, the 3D model representation (i.e. geometry) must allow the calculation of data and comparison with actual measurements (e.g. density variations are measured in Earth's gravity field, variations in magnetization in the magnetic field). Different geometric representations are available in commercial and open source software which supports: (1) layers, horizons, grids, (2) voxel, and (3) polyhedra or triangulated hulls.

\subsubsection{Software}

The decision on which software to use depends on the availability and areal coverage of data and additional information that should be integrated, as well as the target which is going to be modelled. Not every geometry is equally suited to model e.g. distinct ore bodies, salt domes, faults, break-ups, pinch out of layers, and so on and the different geometries offer different possibilities for model modifications and editing.

One of the main interests for the geophysics team at NGU is the structure of the Norwegian shelf and margin. NGU has a wealth of airborne and shipborne potential field data (gravity and magnetic measurements), as well as access to high-resolution seismic data. These data are used complementary. 3D seismic acquisition is expensive and therefore often only done along $2 \mathrm{D}$ profiles across "interesting" structures. Potential field data is cheaper and acquired over large areas. It helps to extent interpretations beyond the seismic lines. Figures 5-7 shows the same model, a cut-out of a lithospheric density model extending from mainland Norway to the Mid-Atlantic ridge, in the three geometric representations that were mentioned above.

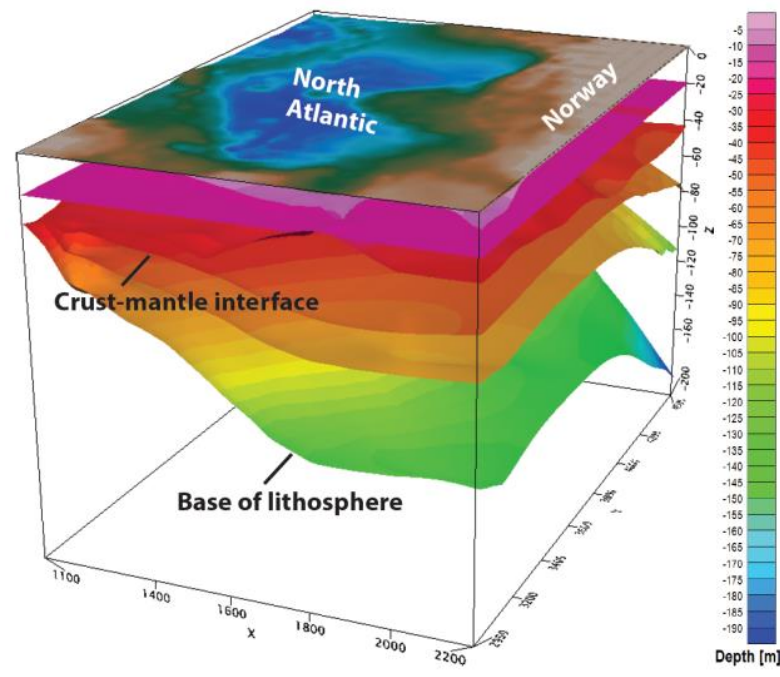

Figure 5: Model in GM-SYS 3D. Displayed are the depth horizons where density contrasts occur.

Figure 5 shows a grid-based model in GM-SYS 3D where interfaces between structural layers are shown. Physical properties (e.g. densities, seismic velocities) are assigned to the spaces between the individual horizons. They can be constant or following lateral or vertical distributions. Modifications of the model geometry are basically limited to mathematical grid operations. Modelling of isolated bodies is difficult.

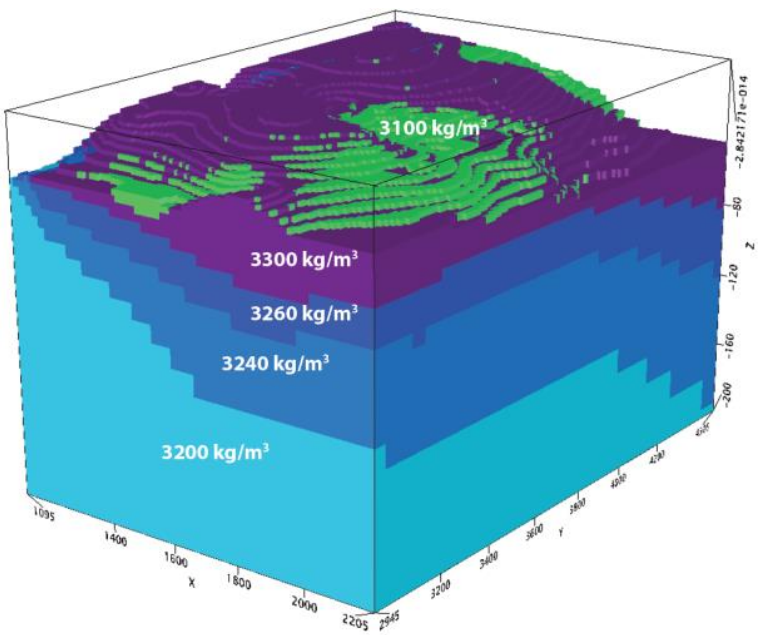

Figure 6: Voxel model in Geosoft. Colours indicate different density values. Voxel sizes are increasing with depth.

In Figure 6 the same model is defined by voxels in Geosoft. All voxels have their assigned physical property and they can be equally sized or increase with depth. The latter is shown here and often recommended in geophysical modelling due to loss of resolution with depth in general and for reduction of model parameters. Voxel models are very handy for imaging of isolated bodies but basically allow any geometry. Furthermore, it is a representation used for several geophysical methods and combinations of these models are more straight forward. It is also a representation often used for the application of mathematical inversion methods and finite-element simulations. The model resolution can be as detailed as computation power and reasonability allows. 


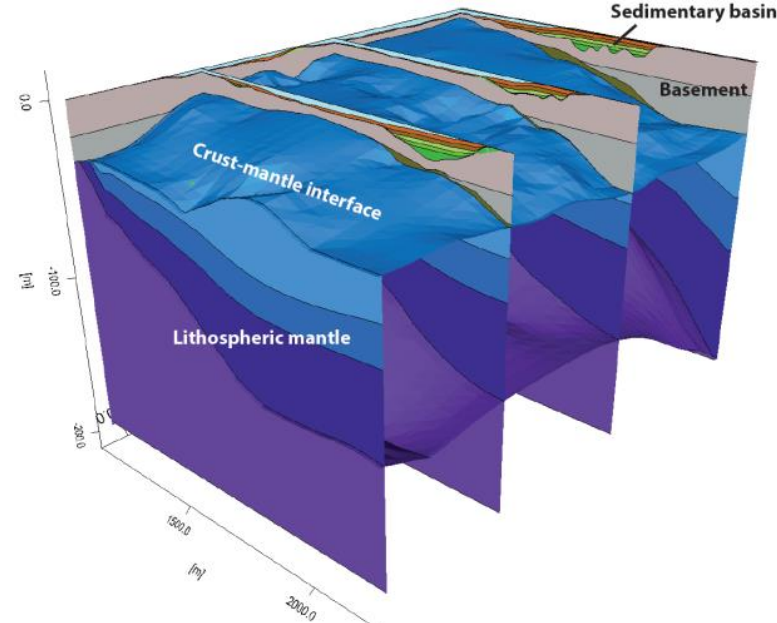

Figure 7: Model of triangulated polyhedron hulls in IGMAS+. Vertical sections and interfaces can be selected as convenient and colours are chosen to follow the international stratigraphy chart.

The third option is given in Figure 7 where the model is built by triangulated polyhedron hulls using IGMAS+. This looks similar to the grid-based model but offers more editing options. The model is actually defined along $2 \mathrm{D}$ vertical sections and then triangulated between these sections to create real 3D bodies. Properties are assigned to the polyhedrons and constant within. Models can be directly modified on the 2D sections and the (de-)selection of individual interfaces and sections allows better visualization for investigations and interpretations. Isolated bodies can be modelled as well as layered structures or faults. Again, the model resolution can be as detailed as computation power and the willingness for manual editing permits.

The mentioned software packages allow more modelling options then described here but the main challenges remain: Editing and visualization of the models and the integration of supplementary data (e.g. information from boreholes, geological models, other geophysical models). We often need to jump between at least 2 modelling applications to perform our desired modifications and handling of the models.

In the example given here, the main interest in the 3D model lies mostly in two of its horizons. One is the crust-mantle boundary and the second is the top of the crustal basement. A detailed knowledge of the latter is important for exploration industry as it describes the bottom of the sedimentary layers (and sedimentary basins), where oil and gas reservoirs are found. The entire model helps in the understanding of the tectonic development of the north Atlantic.

\subsection{Urban geology 3D subsurface modelling in urban areas}

Traditionally, Geological Surveys produce maps for non-urban areas. However, more than $50 \%$ of the world's population live in urban areas and this proportion is expected to increase rapidly in the coming years. Correspondingly, the urban subsurface is being used more and more intensely. Nevertheless, good and readily available geological maps for decision making are scarce, and the importance of the underground tends to be overlooked in city planning. The shallow urban subsurface is typically characterized by a heterogeneous layer of man-made ground that incorporates a wide variety of underground infrastructure, such as pipes, cables, underground parking houses and deep energy wells (Figure 8 and 9). The complexity of the urban subsurface, the need for an efficient use of the available space, as well as the challenge to integrate conflicting interests and requirements, are calling for 3D urban geological mapping and planning.
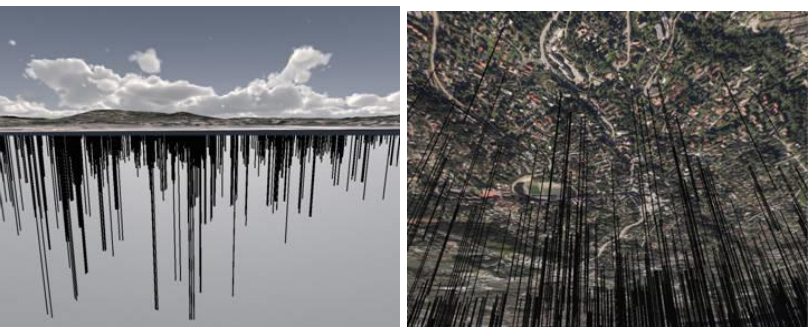

Figure 8: Left: The "Swiss cheese" of energy wells underneath Oslo city (A. Seither).Right: Energy wells underneath Oslo complicate the planning of new infrastructure, such as tunnels (A. Seither)

\subsubsection{D subsurface modelling and visualisation workflow} The World Heritage Site Bryggen in Bergen is one of Norway`s most important historical monuments and a popular tourist destination. Deposits of archaeological value underneath the medieval city centre make for very difficult ground conditions and challenge hereby the development of the city. In a pilot project together with the municipalities of Bergen and the Directorate for Cultural Heritage, the Geological Survey of Norway aims to develop a 3D subsurface modelling and visualisation workflow. This requires adequate data on the geometry and properties (geotechnical, hydrogeological) of both natural and anthropogenic deposits. So far however, the data needed to make an integral analysis are not readily available. There is a large body of existing data but they are stored at different institutions and in an array of different formats. Accordingly, the first step of the project is to systemize these data and to make them interoperable by storing them in one central database (NADAG). The data and the models will form the basis for evaluation of stability and risk analyses, concerning urban development and consequences for groundwater, subsidence and preservation of cultural heritage.

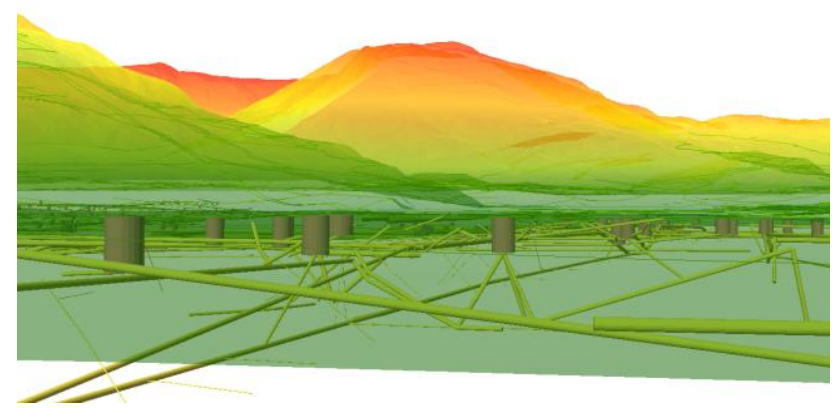

Figure 9: The "Swiss cheese" of energy wells (A. Seither).

\subsection{LiDAR data}

Digital elevation models derived from airborne LiDAR data have increasingly been implemented at NGU, for instance in connection with mapping of surficial deposits. The data render development of detailed and vegetation-free DEMs possible, which subsequently can be utilized to illustrate the terrain, e.g. 
through slope representations and colored and shaded relief images. The $\mathrm{x}, \mathrm{y}, \mathrm{z}$ data also form a valuable foundation when developing precise terrain transects, as illustrated in Figure 10, and when analyzing different terrain parameters.

The accuracy of the LiDAR data depends on the amount of points $/ \mathrm{m}^{2}$, as height data is usually binned or triangulated between the nearest available LiDAR return points when they are processed into a digital elevation model.

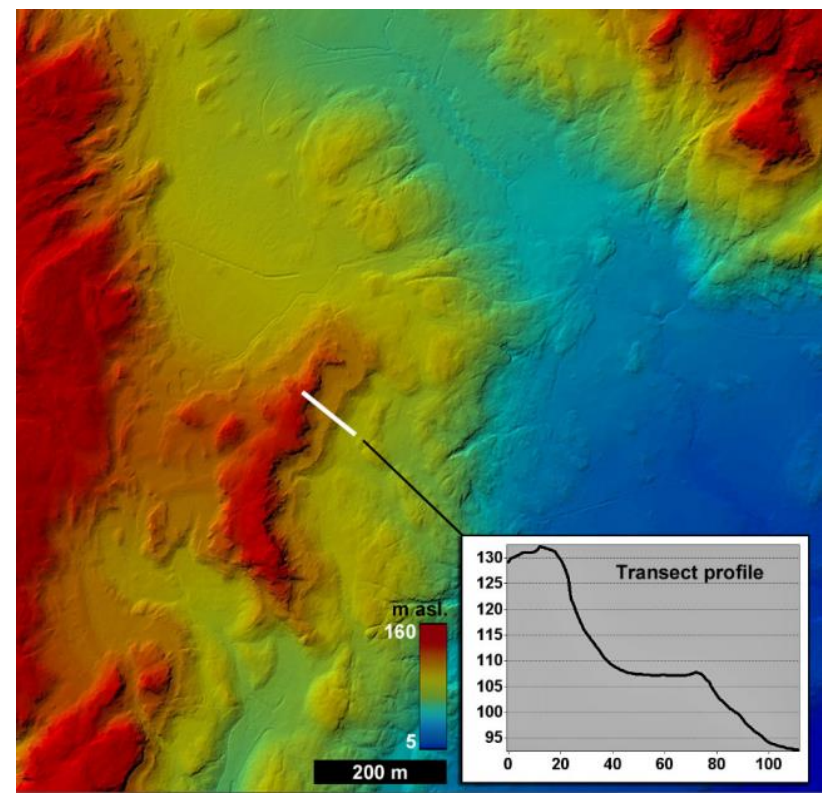

Figure 10: Shoreline morphology. Shaded relief LiDAR image showing the Main Line as a marked discontinuous terrace carved into bedrock (Høgaas, Sveian 2015).

\subsection{WebGL facilitates accessibility of 3D geology}

The WebGL API is a cross-platform, royalty-free API used to create 3D graphics in a Web browser. It runs in the HTML5 Canvas element and has full integration with all Document Object Model (DOM) interfaces, which means that it can be used from any DOM-compatible language like JavaScript. Major Browser vendors are supporting WebGL and are member of the WebGL Working Group. WebGL is based on OpenGL which are still used in a variety of plug-ins. The advantage of WebGL is that there is no need to build a plug-in to use it; is rather to incorporate preferred implementation in JavaScript which runs directly in used browser. The EbGL API is well documented (WebGL 2015).

\subsubsection{Open Source JavaScript frameworks}

To make it easier it for developers, several Open Source JavaScript frameworks has been developed such as theejs (Three.js 2015) and Cesium (AGI 2015). Cesium is supposed to be a coupled part of the OpenLayers3 (OpenLayers 3 2015) JavaScript framework, which is the key framework in NGU's map applications, but the coupling is not in a mature state yet. Inspired by an implementation done at the Norwegian Mapping Athority (Kartverket 2015) with threejs, was started our own implementation in threejs for geological maps and geological profiles (Iversen 2015).

\subsubsection{The implementation needs the following major} input:

- A composite map image (multiple WMS-calls) or a static image.

An elevation grid is delivered by a WCS provided by the Norwegian Mapping Athority (Open Geospatial Consortium 2015), The WCS delivers geotiff or XYZ with elevation data. Geotiff is compact and is quickly transferred.

- A TIN model is generated from the elevation grid.

- The Map image is draped on the TINs surface and we end up with an interactive "Scene". Figure 11 and 12,

\subsubsection{Customisations and methods:}

- The Model (canvas) always adapts to the window size (responsive).

- The pivot point in the model is adjusted to the computed average height to make it easier to tilt.

- Heights must be exaggerated in maps covering larger areas or in areas with smaller height differences.

- A standard height cell represents $8 \times 8$ pixels in the map image.

- The number of pixels pr height cell for large scale maps (small areas) has to be increased to match the actual height resolution which is maximum $10 \mathrm{~m}$. This will remove the stairs effect.

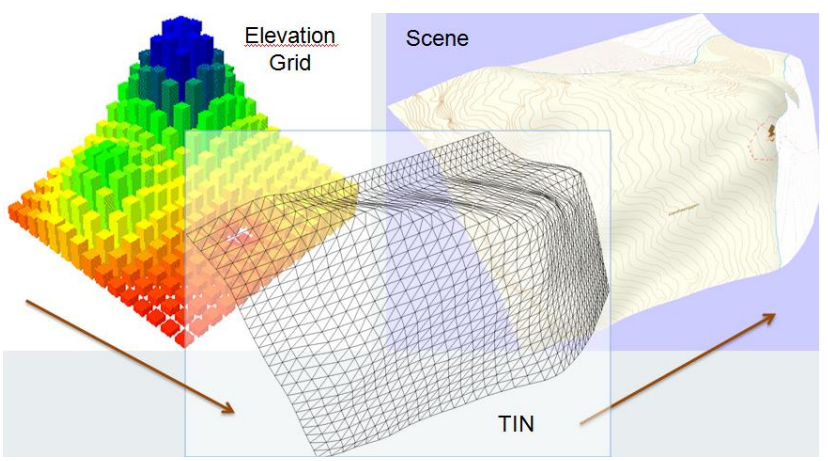

Figure 11: Combination of a TIN and a Map image makes an interactive "Scene".

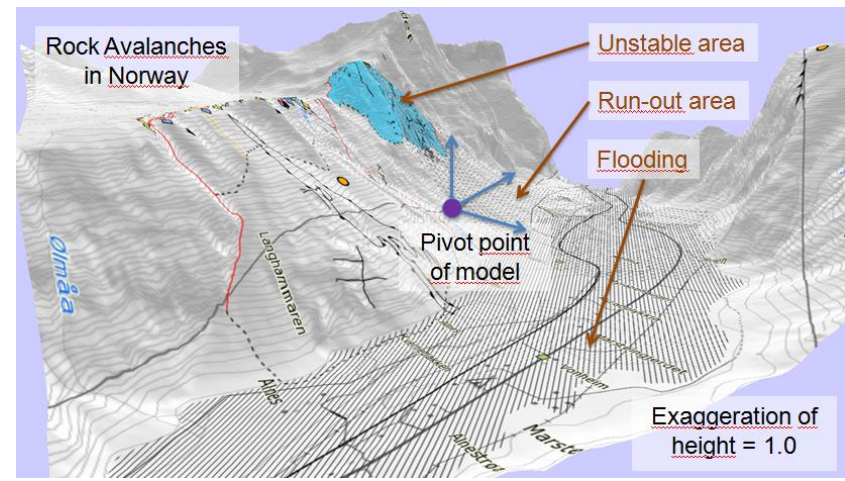

Figure 12: Unstable rock slopes at mountain "Mannen".

\subsubsection{Geological Profiles}

Hundreds of geological profiles exist in numerous maps produced over the year at the Geological Survey of Norway (Figure 13) The work of preparing the profiles for digital use in 3D has started: 
1. Scanning profiles, make them partly transparent.

2. Establish profile Metadata to enable use in a 3D space.

3. To do: build a database for profiles and connection metadata to ease access from different tools and to automate display of profiles in a selected area.

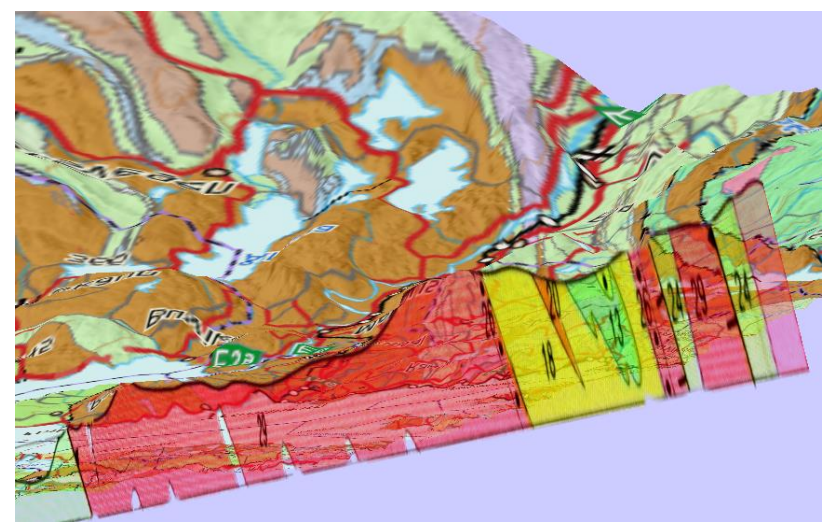

Figure 13: A geological profile projected into 3D space.

\section{DISCUSSION}

Three-dimensional geological modelling and mapping is becoming more popular, and an easier way to understand techniques of portraying geological data. 3D interpretation of geological information can help imagine geological conditions in space, increase interest in geology, and understand problems that are often of high complexity.

As the presented examples demonstrates, 3D activities now are present through the whole chain of management and communication of geological data. There is a range of approaches, methods and software being used. There is work to be done on developing the data management framework for 3D data and models.

The huge variety of available software for creating 3D models allows mapping communities to choose tools which meet their needs. However, the wide variety of approaches to geological modelling and visualization makes it very hard to find standard methods and tools that fulfils all needs at the current stage. Current strategy is to open for development in different fields by delimiting the restrains on methodology, software choices and formats used. At the same time the geomatics department involves more to contribute to good practice and to facilitate access to data and models both internally and externally.

\section{CONCLUSION}

Three-dimensional models provide better communication between industry and local governments, based on better visual information of geological information.

There are various aspects of 3D representation of geological models depending on the data usage: base map, stratigraphic schemes, geophysics, seismic profiles, nationwide dip and strike, data scales or resolutions of models.

The IT and Geomatics division at the Geological Survey of Norway aims to facilitate the standardisation, systematisation, management of the 3D data and models, and has begun to assess the possibilities for a web-based user interface.

\section{REFERENCES}

AGI, 2015-last update, Celsium. Available: http://cesiumjs.org/ [06, 2015].

HØGAAS, F. and SVEIAN, H., 2015. The Younger Dryas Main Line on Leka, Norway, as determined from a high resolution digital elevation model derived from airborne LiDAR data. Geomorphology, 231(0), pp. 63-71.

IVERSEN, S., 2015-last update, Sverre-Iversen/wxs.threejs. Available: https://github.com/Sverre-Iversen/wxs.threejs [07, 2015].

KARTVERKET, 07, 2015-last update, WCS i threejs. Available: http://labs.kartverket.no/wcs-i-threejs/ [07, 2015].

OPEN GEOSPATIAL CONSORTIUM, 2015-last update, Web Coverage Service. Available:

http://www.opengeospatial.org/standards/wcs [06, 2015].

OPENLAYERS 3, 2015-last update, OpenLayers 3. Available: http://openlayers.org [05, 2015].

THREE.JS, 2015-last update, Three.js. Available: http://threejs.org/ [07, 2015].

WEBGL, 2015-last update, Main Page. Available: https://www.khronos.org/webgl/wiki/Main_Page [05, 2015].

WIJNS, C., BOSCHETTI, F. and MORESI, L., 2003. Inverse modelling in geology by interactive evolutionary computation. Journal of Structural Geology, 25(10), pp. 1615-1621.

WU, Q., XU, H. and ZOU, X., 2005. An effective method for 3D geological modeling with multi-source data integration. Computers \& Geosciences, 31(1), pp. 35-43. 